\title{
Identification of Functional Circuitry between Retrosplenial and Postrhinal Cortices during Fear Conditioning
}

\author{
Siobhan Robinson, Caroline E. Poorman, Thomas J. Marder, and David J. Bucci \\ Department of Psychological and Brain Sciences, Dartmouth College, Hanover, New Hampshire 03755
}

\begin{abstract}
The retrosplenial cortex (RSP) and postrhinal cortex (POR) are heavily interconnected with medial temporal lobe structures involved in learning and memory. Previous studies indicate that RSP and POR are necessary for contextual fear conditioning, but it remains unclear whether these regions contribute individually or instead work together as a functional circuit to modulate learning and/or memory. In Experiment 1, learning-related neuronal activity was assessed in RSP from home cage, shock-only, context-only, or fear-conditioned rats using real-time PCR and immunohistochemical methods to quantify immediate-early gene expression. A significant increase in activityregulated cytoskeleton-associated protein ( $A r c)$ mRNA and Arc and c-Fos protein expression was detected in RSP from fear-conditioned rats compared with all other groups. In Experiment 2, retrograde tracing combined with immunohistochemistry revealed that, compared with controls, a significant proportion of cells projecting from RSP to POR were immunopositive for c-Fos in fear-conditioned rats. These results demonstrate that neurons projecting from RSP to POR are indeed active during fear conditioning. In Experiment 3, a functional disconnection paradigm was used to further examine the interaction between RSP and POR during fear conditioning. Compared with controls, rats with unilateral lesions of RSP and POR on opposite sides of the brain exhibited impaired contextual fear memory, whereas rats with unilateral lesions in the same hemisphere displayed intermediate levels of freezing compared with controls and rats with contralateral lesions. Collectively, these results are the first to show that RSP and POR function as a cortical network necessary for contextual fear learning and memory.
\end{abstract}

\section{Introduction}

The retrosplenial cortex (RSP) is highly interconnected with parahippocampal regions, providing a major source of visuospatial sensory information to the hippocampal-parahippocampal network in primates and rodents (Fig. 1) (Burwell and Amaral, 1998a,b; Kobayashi and Amaral, 2007). Through connections with areas such as postrhinal cortex (POR), RSP is positioned to contribute to hippocampal-dependent functions including spatial and contextual learning and memory (Burgess, 2008; Sugar et al., 2011). Consistent with this circuitry, studies in humans indicate that both RSP and parahippocampal cortex (comparable to POR in rats) are activated during the processing of highly contextual objects but not during the processing of objects with weak contextual associations (Bar and Aminoff, 2003; Staresina et al., 2011). Similarly, damage to either RSP or POR impairs contextual fear conditioning in rats. In a typical fear-conditioning paradigm, a tone is paired with footshock during training and subsequent reexposure to either the training context or the tone

\footnotetext{
Received June 13, 2012; revised July 5, 2012; accepted July 12, 2012.

Author contributions:S.R. and D.J.B. designed research;S.R., C.E.P. and T.J.M. performed research; S.R. analyzed data; S.R. and D.J.B. wrote the paper.

This work was supported by National Science Foundation Grant 0922075 (D.J.B.) and National Institutes of Health Grant MH092991 (S.R.). We thank Dr. Joyce A. DeLeo for her intellectual contributions to this project and Kenneth Orndorff for assistance with the confocal microscopy experiment. We also thank Dr. Robert Leaton and two anonymous reviewers for valuable comments on a previous version of this manuscript.

The authors declare no competing financial interests.

Correspondence should be addressed to Dr. Siobhan Robinson, Dartmouth College, 6207 Moore Hall, Hanover, NH 03755. E-mail: siobhan.robinson@dartmouth.edu.

DOI:10.1523/JNEUROSCI.2814-12.2012

Copyright $\odot 2012$ the authors $\quad 0270-6474 / 12 / 3212076-11 \$ 15.00 / 0$
}

elicits a conditioned fear response in normal rats (Fanselow, 1980; Maren and Fanselow, 1997). Lesions of either RSP or POR that occur either before or after training impair the expression of contextual fear but not tone-specific fear (Bucci et al., 2000; Keene and Bucci, $2008 \mathrm{a}, \mathrm{c})$, supporting the notion that RSP and POR contribute to forming configural associations between multiple sensory stimuli that compose the training environment (Maren et al., 1998; Holland and Bouton, 1999).

Nevertheless, the specific contributions of individual areas, such as RSP, to learning and memory are currently the focus of much research and debate (Vann et al., 2009; Aggleton, 2011; Eichenbaum et al., 2011; Wixted and Squire, 2011). For example, current support for the view that RSP has a role in learning about associations between multiple sensory stimuli is provided primarily by lesion studies (Harker and Whishaw, 2004; Smith et al., 2004; Talk et al., 2005; Keene and Bucci, 2008b; Robinson et al., 2011). However, it is often difficult to distinguish between lesion effects on learning versus memory; moreover, no studies have examined whether RSP neurons are active during acquisition of contextual fear conditioning. Additionally, it is unclear whether RSP and POR form a functional circuit that supports contextual learning and/or memory or whether these regions function in a more independent fashion (Vann et al., 2009; van Strien et al., 2009; Sugar et al., 2011). Indeed, while previous studies provide evidence for a functional relationship between RSP and the hippocampus (Cooper and Mizumori, 2001; Kubik et al., 2012), no study to date has demonstrated a functional connection between RSP and POR during contextual learning/memory. To address these questions, the present study used a combination of ap- 


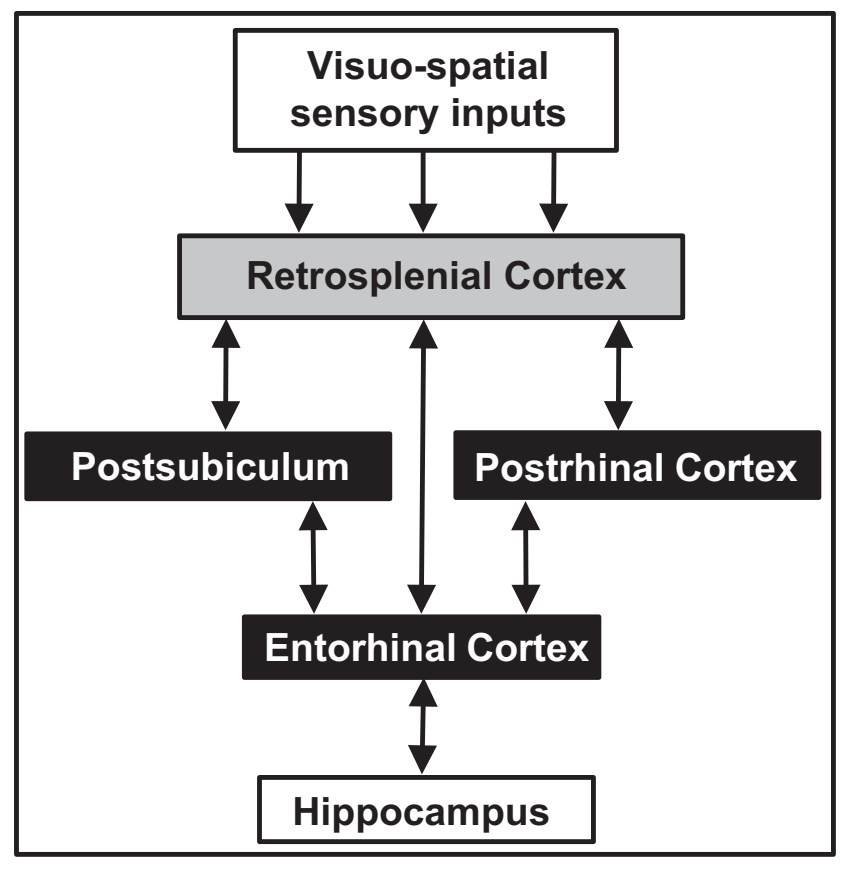

Figure 1. Schematic of corticohippocampal circuitry depicting three pathways from RSP to the hippocampus.

proaches including analysis of neural activity, anatomical tracing, and lesion disconnection methods. First, we tested whether RSP neurons are active during the acquisition of fear conditioning by quantifying immediate-early gene (IEG) expression directly after acquisition of fear conditioning. A second set of experiments investigated the neural circuitry underlying learning and memory by testing the hypothesis that communication between RSP and POR is critical for fear conditioning.

\section{Materials and Methods Experiment 1 \\ Experimental design}

To determine whether RSP neurons are active during acquisition of contextual fear conditioning, IEG expression was quantified across four behavioral conditions. In the "paired" condition, rats received standard context-footshock pairings; rats in the "context-only" group were placed in the training chamber, but no shock was delivered; rats in the "shockonly" group received footshocks that were not associated with the chamber; and rats in the "home cage" group did not experience the training chamber or any footshocks. The transcription factor c-Fos was selected as a marker of neuronal activity because it has been used to assess regionspecific activity during fear conditioning in previous studies (Campeau et al., 1991; Pezzone et al., 1992; Beck and Fibiger, 1995). In addition, expression of Arc was assessed because it has been specifically linked to activity-dependent synaptic plasticity (Miyashita et al., 2009; Shepherd and Bear, 2011), and prior studies have demonstrated context-specific expression of Arc in hippocampal and parahippocampal regions (Guzowski et al., 1999; Vazdarjanova et al., 2006). Recent work has also shown that Arc expression is elevated in RSP during hippocampaldependent learning (Tse et al., 2011).

\section{Subjects}

Naive male Long-Evans rats (weighing 225-250 g upon arrival) were obtained from Harlan Laboratories and housed singly in a temperaturecontrolled room with a 14:10 h light/dark cycle with lights on at 7:00 A.M. Rats had ad libitum access to food (Purina standard rat chow: Nestle Purina) and water. Rats were handled for $2 \mathrm{~min}$ per day for $3 \mathrm{~d}$ before behavioral testing. All efforts were made to minimize discomfort and to limit the number of rats used. The Institutional Animal Care and Use
Committee at Dartmouth College approved the use of rats in these studies, and all procedures were conducted in accordance with $\mathrm{NIH}$ guidelines.

\section{Behavioral apparatus}

Fear conditioning was conducted in standard operant conditioning chambers $(24 \times 30.5 \times 29 \mathrm{~cm}$; MED Associates $)$ connected to a computer and enclosed in sound-attenuating chambers $(62 \times 56 \times 56 \mathrm{~cm})$ outfitted with an exhaust fan to provide airflow and background noise $(\sim 68 \mathrm{~dB})$. The operant chambers consisted of aluminum front and back walls, clear acrylic sides and top, and grid floors. A food cup was recessed in the center of the front wall $(5 \mathrm{~cm}$ above the floor), and a jeweled panel light was located $15 \mathrm{~cm}$ above the opening of the recessed food cup (not used in this study). A $6 \mathrm{~W}$ house light providing background illumination was mounted near the ceiling on the opposite wall. A speaker was located $15 \mathrm{~cm}$ above and to the right of the food cup. Delivery of a $1.0 \mathrm{~mA}, 1.0 \mathrm{~s}$ constant-current shock through the grid floor of the operant chamber served as the unconditioned stimulus. Surveillance cameras located inside the sound-attenuating chambers were used to videorecord the rats' behavior.

\section{Behavioral treatment groups}

Three cohorts of rats were behaviorally trained: rats in the first cohort $(n=15)$ were used to confirm the immediate shock deficit effect (described below), rats in the second cohort $(n=26)$ were used for the immunohistochemistry (IHC) experiment, and rats in the third cohort $(n=24)$ were used for the quantitative real-time reverse transcriptase PCR (qRT-PCR) experiment. A schematic diagram of the behavioral conditions (home cage, shock-only, context-only, and paired) is shown in Figure $2 \mathrm{~A}$. On the training day, rats in the paired group were placed in the training chamber and were permitted to explore the environment for $3 \mathrm{~min}$. Subsequently, three $1.0 \mathrm{~s}, 1.0 \mathrm{~mA}$ footshocks were administered with an intertrial interval (ITI) of $64 \mathrm{~s}$. Rats were removed from the chambers $64 \mathrm{~s}$ after the last footshock. Rats in the context-only group were placed in the conditioning chamber for the same amount of time as the paired group, but no shocks were administered. Rats in the shockonly group were placed in the chamber with no illumination and immediately administered a series of three $1.0 \mathrm{~s}, 1.0 \mathrm{~mA}$ footshocks (ITI of $2 \mathrm{~s}$ ) and then removed immediately from the chamber. The fourth group of rats was not exposed to the chambers and remained in their home cages. The context-only group served as a control for context-induced changes in gene expression in the absence of shock. This group is an essential control given the context-specific expression of Arc in hippocampal and parahippocampal regions (Guzowski et al., 1999). Inclusion of the shock-only group controlled for shock-induced changes in gene expression in the absence of a context-shock association.

Upon completion of training, rats in cohort 1 were tested for fear memory (described below). Rats in cohort 2 in the shock-only $(n=6)$, context-only $(n=7)$, and paired $(n=6)$ groups were removed from the conditioning chambers and returned to their home cages for $60 \mathrm{~min}$ after which they were deeply anesthetized with an overdose of pentobarbital sodium and phenytoin sodium (Euthasol; Virbac Animal Health) and transcardially perfused with $0.9 \%$ saline for $5 \mathrm{~min}$, followed by $4 \%$ paraformaldehyde in $0.1 \mathrm{M}$ PBS. Rats in the home cage control group $(n=7)$ were briefly removed from their home cages, injected with euthasol and immediately returned to their home cages until deeply sedated after which they were transcardially perfused. The $60 \mathrm{~min}$ time point for the protein assays was selected based on reports of Arc expression measured $\sim 1 \mathrm{~h}$ after exposure to a novel environment in the rodent hippocampus (Vazdarjanova et al., 2006) and in murine RSP tissue (Spulber et al., 2009). Similarly, the level of c-Fos protein has been shown to peak $60 \mathrm{~min}$ after induction (Hughes and Dragunow, 1994). Rats in cohort 3 in the shock-only $(n=6)$, context-only $(n=5)$, and paired group $(n=7)$ were removed from the conditioning chambers and returned to their home cages for $30 \mathrm{~min}$ after which they were sedated with carbon dioxide gas and killed by rapid decapitation. Home cage control rats $(n=6)$ were similarly sedated and decapitated. The $30 \mathrm{~min}$ time point for the mRNA study was selected based on previous time course studies revealing robust hippocampal Arc mRNA expression 15-45 min after exploration of a 
novel environment (Guzowski et al., 1999) and peak Arc mRNA expression in the amygdala 30 min after fear conditioning (Ploski et al., 2008).

\section{Immediate shock deficit procedure}

The protocol described above for the paired group is known to reliably support contextual fear conditioning (Maren and Fanselow, 1997; Maren et al., 1997; Arenos et al., 2006; Keene and Bucci, 2008c), whereas the protocol used for the shock-only group results in the immediate shock deficit phenomenon in which shock-only rats do not express fear memory (i.e., they do not freeze) during a subsequent test for contextual fear memory (Fanselow, 1980, 1986). To confirm that a shock-context association did not occur in rats in the shock-only group, rats from cohort 1 in the paired group $(n=7)$ and shock-only $(n=8)$ groups were reexposed to the chamber $24 \mathrm{~h}$ after the training session for a 10 min context test during which no shocks were presented. Behavior was videorecorded throughout.

\section{Behavioral observations}

Freezing served as the index of conditioned fear and was operationally defined as total motor immobility except for breathing (Blanchard and Blanchard, 1969; Fanselow, 1980). For the training day data, the incidence of freezing behavior was scored during the $64 \mathrm{~s}$ epoch before the first trial (baseline freezing) and during the $64 \mathrm{~s}$ periods following each footshock (i.e., postshock freezing). The rats' behavior was recorded every $8 \mathrm{~s}$ during the $64 \mathrm{~s}$ epochs. The context test session was also divided into $64 \mathrm{~s}$ epochs and behavior was scored every $8 \mathrm{~s}$. The frequency of freezing behavior was converted to a percentage of total observations. A primary observer scored the behavioral data, while two additional observers scored a subset of the data to assess objectivity. All observers were blind to treatment condition and their observations were highly correlated $(r=0.92 ; p<0.001)$.

\section{Immunohistochemistry}

Brain sections from rats in cohort 2 were assessed for c-Fos and Arc protein expression. Upon removal, brains were placed in fixative overnight and subsequently placed in a solution of $30 \%$ sucrose in PBS for $4 \mathrm{~d}$ and frozen at $-80^{\circ} \mathrm{C}$ until sectioning. Free-floating sections $(40 \mu \mathrm{m})$ containing RSP [ $\sim 3.5$ and $5.0 \mathrm{~mm}$ posterior to bregma according to the rat brain atlas of Paxinos and Watson (2007)] were cut with a freezing microtome and incubated in $0.3 \%$ hydrogen peroxide in PBS for $10 \mathrm{~min}$, rinsed, and then blocked for $1 \mathrm{~h}$ in immunobuffer (IB) containing $2 \%$ normal goat serum and $0.1 \%$ Triton X-100 (Sigma-Aldrich) in PBS. Sections were then incubated overnight at $4^{\circ} \mathrm{C}$ in IB containing primary rabbit polyclonal c-Fos antibody (Santa Cruz Biotechnology; sc-52; lot no. C1110; 1:10,000 dilution). After rinses in PBS, sections were incubated for $2 \mathrm{~h}$ in biotinylated goat anti-rabbit secondary IgG (Vector Laboratories), rinsed, and incubated for $30 \mathrm{~min}$ in avidin-biotin complex (ABC Vectastain Kit; Vector). Chromagen immunostaining was visualized with using a diaminobenzidine kit (Vector). In addition, region-matched tissue from a subset of rats ( $n=4$ /group) were assayed for Arc protein expression using the same procedure with a primary rabbit polyclonal Arc antibody (Santa Cruz Biotechnology; sc-15325; lot no. G3009; 1:500 dilution).

\section{Microscopy and cell counting}

RSP sections were slide-mounted and examined by an observer blind to the behavioral condition using an Axioskope I microscope (Zeiss) connected to a computer equipped with StereoInvestigator software (version 9.0; MicroBrightField). IEG-positive cells in RSP (see Fig. $2 D$, shaded region of the brain diagrams) were counted from either the left or right hemisphere from two region-matched sections from each rat. A contour was drawn at $2.5 \times$ magnification to outline the boundaries of RSP according to the rat brain atlas of Paxinos and Watson (2007). Cell counting was then conducted at $20 \times$ magnification using the MeanderScan tool in StereoInvestigator, in which a counting frame $(0.3 \times 0.35 \mathrm{~mm})$ was placed within the boundaries of the target area and advanced systematically through the region. The number of c-Fos- or Arc-positive cells in a counting frame was determined using a planometric analysis in which an optical density threshold of no less than $33 \%$ of the surrounding background stain was set for counting a cell as immunopositive. Because sections were $1500 \mu \mathrm{m}$ apart, it was not necessary to correct for double counting.

\section{$R N A$ isolation and quantitative real-time PCR}

RSP tissue from rats in cohort 3 was assessed for relative Arc mRNA expression. Brains were removed, coated with RNAlater (QIAGEN), and immediately frozen in crushed dry ice for $2 \mathrm{~min}$. RSP was dissected (the rostral cut was made $7 \mathrm{~mm}$ from the front of the brain, a second pair of cuts was made $2 \mathrm{~mm}$ lateral to the midline on each hemisphere, a third cut was made $2 \mathrm{~mm}$ ventral from the surface of the brain, and last, cerebellar tissue was removed from the caudal end of the RSP tissue), transferred to ice-cold microcentrifuge tubes, and stored at -80 until RNA isolation. Total RNA was isolated from RSP tissue using the RNeasy Lipid Tissue Kit (QIAGEN), and $1 \mu \mathrm{g}$ of each sample was reversetranscribed according to the vendor's suggested protocol in the QuantiTect Reverse Transcription Kit (QIAGEN). qRT-PCR was performed using the $\Delta \Delta$ Ct method with a 7500 Real-Time PCR System (Applied Biosystems). The DNA sequences of rat glyceraldehyde 3-phosphate dehydrogenase (GAPDH) and ARC were obtained from GenBank. Forward and reverse primers and TaqMan probes (assay IDs: Rn00571208_g1 and Rn01775763_g1 for Arc and GAPDH, respectively) were purchased from Applied Biosystems. The TaqMan probes have a fluorescent reporter dye, FAM (6-carboxyfluorescein), at the $5^{\prime}$ end; this fluorescence, which is directly proportional to the PCR product, is continually monitored during the real-time PCR. All samples were run in triplicate, and each sample was normalized against GAPDH levels from the same sample. Total RNA was included in each run as a negative control. Data from rats in the shock-only, context-only, and paired groups were normalized to the average value of home cage controls.

\section{Data analysis}

Behavior. Freezing data collected during the context test were subjected to a repeated-measures ANOVA with Group (Shock-only, Paired) as the between-subjects variable and Block (64 s epoch) as the within-subjects variable. Data from the training session for rats in the paired group were subjected to repeated-measures ANOVA using Trial (baseline and postshock 1,2, and 3) as the within-subjects variable. Freezing behavior displayed by the shock-only group during conditioning was not analyzed because training took place in the dark and there were $2 \mathrm{~s}$ ITIs instead of 64 s ITIs.

Neurochemistry. To analyze IHC data, c-Fos-positive cell counts collected from the two RSP sections from each rat were averaged to produce a single value and then one-way ANOVA was performed on data from the four (home cage, shock-only, context-only, and paired) behavioral groups. Arc-positive cell counts were analyzed similarly. To analyze qRTPCR data, relative Arc mRNA levels from RSP tissue were subject to one-way ANOVA. An $\alpha$ value of 0.05 was adopted for all analyses, and post hoc analyses using Fisher's least significant difference test were used when appropriate.

\section{Experiment 2}

\section{Experimental design}

Although neuroanatomical studies have established that POR receives significant input from RSP (Burwell and Amaral, 1998a,b; Sugar et al., 2011), it is currently unknown whether the connection between these regions is active when processing contextual information. This was tested in Experiment 2 by infusing a retrograde tracer into POR before training in the contextual fear-conditioning task. After training, brain tissue was processed for c-Fos immunohistochemistry. Sections containing RSP were analyzed for the presence of neurons that were double labeled for the retrograde tracer and c-Fos.

\section{Subjects}

Ten Long-Evans rats were obtained and housed as described for Experiment 1 .

\section{Surgery}

Before behavioral testing, rats were anesthetized with isoflurane gas (1.5-3\% in oxygen) and placed in a Kopf stereotaxic apparatus. A solution $(0.5 \% \mathrm{w} / \mathrm{v})$ of cholera toxin subunit B conjugated to Alexa Fluor 488 
(CTb-488) (catalog \#C34775; Invitrogen) was injected (0.2 $\mu \mathrm{l} /$ site at a rate of $0.1 \mu \mathrm{l} / \mathrm{min}$ ) unilaterally into the POR. CTb- 488 was chosen as the retrograde tracer because of the high specificity of transport and the bright label provided by this compound (Conte et al., 2009). In addition, previous studies have shown that detection of fluorescently tagged CTb labeling is compatible with immunohistochemical techniques (Matsui and Williams, 2010). CTb-488 was infused into POR using the following coordinates with respect to lambda (in $\mathrm{mm}$ ): $+0.5,-0.1$ anteroposterior, -0.4 lateral, and $-4.4,-3.8$ ventral from the skull surface, based on previous studies (Bucci et al., 2000; Burwell and Hafeman, 2003) and on the rat brain atlas of Paxinos and Watson (2007). The Hamilton syringe (26 ga) used to deliver the tracer was left in place for $3 \mathrm{~min}$ following each infusion to reduce the diffusion of tracer along the needle tract. Rats were allowed to recover for $10-12 \mathrm{~d}$ before behavioral testing.

\section{Behavioral apparatus and procedure}

The conditioning chambers were identical with those described in Experiment 1 . On the conditioning day, rats in the paired group $(n=6)$ were fear conditioned as described in Experiment 1 and a second group of rats $(n=4)$ served as home cage controls.

\section{Fluorescence immunohistochemistry}

Rats in the paired group were removed from the conditioning chambers and returned to their home cages for $60 \mathrm{~min}$ after which they were deeply anesthetized with an overdose of Euthasol and transcardially perfused with $0.9 \%$ saline for $5 \mathrm{~min}$, followed by $4 \%$ paraformaldehyde in PBS. Home cage controls were likewise deeply anesthetized and then perfused. Brains from rats in the paired and home cage groups were placed in fixative for $45 \mathrm{~min}$ and subsequently placed in a solution of $30 \%$ sucrose in PBS for $4 \mathrm{~d}$. Free-floating sections $(40 \mu \mathrm{m})$ containing RSP were cut with a freezing microtome and incubated in $0.1 \%$ sodium borohydride in PBS for $30 \mathrm{~min}$, rinsed, and then blocked for $1 \mathrm{~h}$ in IB containing 20\% normal goat serum and $0.1 \%$ Triton X-100 in PBS. Sections were then incubated for $72 \mathrm{~h}$ at $4^{\circ} \mathrm{C}$ in primary rabbit polyclonal c-Fos antibody (Santa Cruz Biotechnology; sc-52; lot no. G1911; 1:1000 dilution) in IB containing 2\% normal goat serum. c-Fos was used as the marker of neuronal activation based on the robust expression observed in Experiment 1. Furthermore, because c-Fos expression is nuclear, it is more readily distinguished from the pattern of labeling provided by the tracer (which labels dendrites, axons, and perikaryon). After rinses in PBS, sections were incubated for $4 \mathrm{~h}$ in Alexa 555 goat anti-rabbit secondary IgG (Invitrogen; 1:200 dilution) and Neurotrace 435/455 (Invitrogen; 1:300 dilution), rinsed, slide mounted, and coverslipped with Gelvatol mounting medium.

Microscopy, cell counting, and detection of colocalized fluorescence Slides containing sections with POR were imaged by an experimenter blind to behavioral conditions using a Zeiss fluorescence microscope to verify microinfusion into the POR. In addition, the laterodorsal posterior thalamus, perirhinal cortex, and visual cortex were viewed in each rat as a positive control for accurate infusion placement (Burwell and Amaral, 1998a,b; Furtak et al., 2007). Confocal microscopy was used to quantify cells labeled with the CTb- 488 tracer, c-Fos, and Nissl using an LSM 510 meta laser-scanning system (Zeiss). Sections containing RSP [see Fig. $5 C$; approximately -6.8 to $-7.5 \mathrm{~mm}$ posterior to bregma according to the atlas of Paxinos and Watson (2007)] were selected for analyses based on detailed anatomical descriptions of projections from RSP to POR (Burwell and Amaral, 1998a,b; Furtak et al., 2007; Jones and Witter, 2007). Between three and six sections from each rat were counted from the hemisphere ipsilateral to the infusion site. Between one and five samples from each section were collected using a $63 \times$ oil-immersion objective. All images were captured through the $Z$-plane. The 10 slices through the $Z$-stack were projected onto a single plane, and cells positive for each c-Fos and CTb-488 were counted independently using the particle analysis program from Fiji-NIH software. In addition, the entire data set was recounted using the cell counter plug-in for Fiji-NIH software. Cells identified as coexpressing CTb-488 and c-Fos were further evaluated through all slices in the $Z$-stack, and only CTb-488-positive cells displaying round nuclei were included in the colocalization analyses.

\section{Table 1. Stereotaxic coordinates for unilateral disconnection lesions}

\begin{tabular}{llll}
\hline Lesion site & AP & ML & DV \\
\hline RSP & -2.0 & \pm 0.3 & -1.6 and -2.5 \\
& -3.5 & \pm 0.4 & -1.8 and -2.5 \\
& -5.0 & \pm 0.4 and \pm 1.0 & -1.6 and -2.2 (medial site), -1.6 (lateral site) \\
& -6.5 & \pm 0.8 and \pm 1.4 & -1.6 and -2.2 (medial site), -2.7 (lateral site) \\
& -8.0 & \pm 1.0 and \pm 2.0 & -2.0 (medial site), -2.4 (lateral site) \\
POR & +0.5 & \pm 4.4 & -5.3 \\
& -0.1 & \pm 4.4 & -4.0 \\
\hline
\end{tabular}

Note: Anterior-posterior (AP), medial-lateral (ML), and dorsal-ventral (DV) measurements for RSP lesions are derived from bregma, midline, and skull surface, respectively (measurements are in millimeters). AP measurements for POR lesions are derived from lambda. Electrolytic lesions were made by applying a $2.5 \mathrm{~mA}$ current for $15 \mathrm{~s}$ at each RSP site and for $10 s$ at each POR site. Lesion placement was counterbalanced between the right and left hemispheres across rats. \pm indicates that a lesion was placed on either the left or the right hemisphere.

\section{Data analysis}

To analyze IHC data, c-Fos-positive cell counts were averaged across the sections from each rat to produce a single value for each subject, and then an independent-samples $t$ test was performed on data from paired and home cage groups. An identical analysis of data from the same sections was used to quantify the percentage of cells colabeled with CTb-488 and c-Fos immunofluorescence.

\section{Experiment 3}

\section{Experimental design}

Previous lesion studies indicate that bilateral damage to either RSP or POR impairs the expression of contextual fear memory (Bucci et al., 2000; Keene and Bucci, 2008a,c). However, those deficits could arise from damage to RSP or POR per se, or because RSP and POR were functionally disconnected from each other. In other words, RSP and POR may make individual contributions to contextual fear conditioning or instead form part of a functional circuit. Although the results of Experiment 2 indicate that connections between RSP and POR are active during fear conditioning, those findings alone do not indicate whether that connection is necessary to support contextual learning/memory. This was addressed in Experiment 3 using an established asymmetric lesion approach to test for functional interactions between structures (Olton, 1983; Warburton et al., 2001; Rogers and Kesner, 2007). Before fear conditioning, one set of rats received unilateral lesions of RSP and POR in opposite hemispheres (i.e., right RSP and left POR, or left RSP and right POR), which interrupts communication between RSP and POR on both sides of the brain, resulting in a complete functional disconnection ("contralateral-lesion" group) (CONTRA). A second set of rats received unilateral RSP and POR lesions in the same hemisphere (i.e., left RSP and left POR, or right RSP and right POR), thus leaving communication between RSP and POR intact on one side of the brain ("ipsilateral lesion" group) (IPSI). If communication between RSP and POR is important during contextual fear conditioning, it would be expected that rats in the CONTRA group would be more impaired than rats in the IPSI group since the latter group still had a functional connection on one side of the brain. Moreover, both lesion groups would be expected to differ from a sham-lesioned group with intact connections on both sides of the brain.

\section{Subjects}

Thirty-two Long-Evans rats were obtained and housed as described for Experiment 1.

\section{Surgery}

Rats were anesthetized with isoflurane gas (1.5-3\% in oxygen) and placed in a Kopf stereotaxic apparatus. Fourteen rats received unilateral lesions of RSP and POR in opposite hemispheres (CONTRA group) and 11 rats received unilateral lesions of RSP and POR in the same hemisphere (IPSI group) before behavioral training using the stereotaxic coordinates outlined in Table 1. An electrode that was epoxy-coated except for the tip was lowered into each coordinate, and a $2.5 \mathrm{~mA}$ current was passed through the tip for $10-15 \mathrm{~s}$ per lesion site. For the POR lesions, the electrode was angled at $22^{\circ}$. Electrolytic lesions were used to provide control over the extent of damage, which was an important factor in this study given the close proximity of posterior parietal cortex and perirhinal cortex, which also provide visuospatial input to the medial temporal lobe 

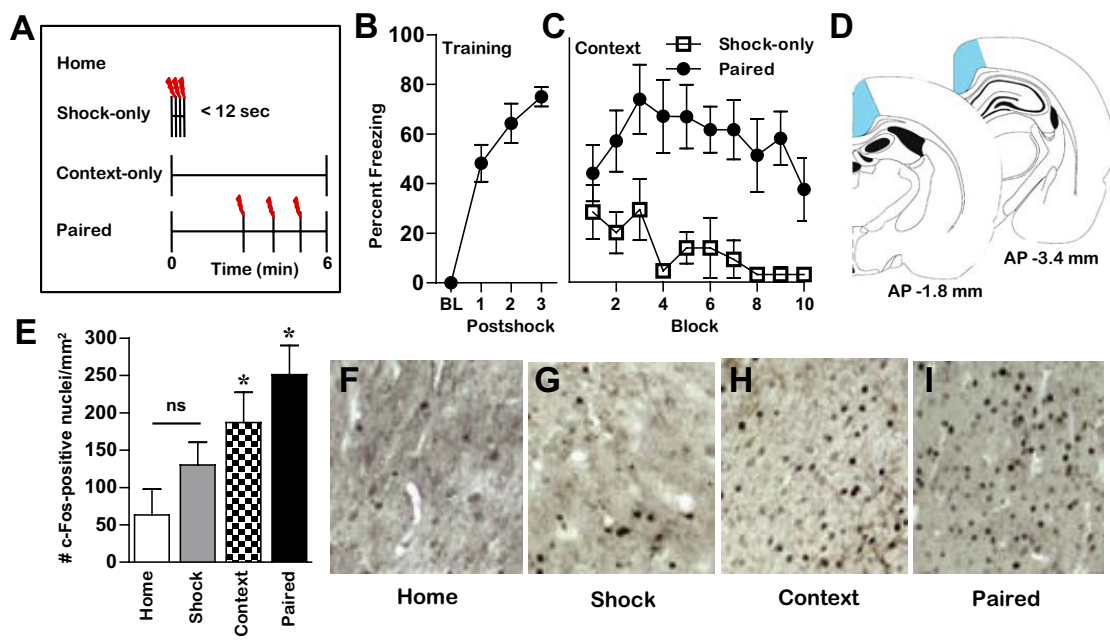

Figure 2. Behavior and c-Fos protein expression in RSP following contextual fear conditioning. A, A schematic depicting the four behavioral conditions included in Experiment 1. Percentage freezing behavior displayed by rats during training (B) and context reexposure ( $\boldsymbol{C}$, confirming that rats in the shock-only condition did not form a context-shock association during acquisition. BL, Baseline. Shock-only $(n=8)$; Paired ( $n=7)$. $\boldsymbol{D}$, Brain diagrams representing the AP location of RSP (shaded area) from which IEG counts were made. The numbers in $\boldsymbol{D}$ reflect the AP distance (in millimeters) from bregma. $E$, Average (mean \pm SEM) c-Fos protein expression in RSP across behavioral conditions. Home, Home cage $(n=7)$; Shock, shock-only $(n=6)$; Context, context-only $(n=7)$; Paired $(n=6)$. $\boldsymbol{F}-\mathbf{I}$, Representative images of c-Fos expression in RSP across behavioral conditions. The asterisk $(*)$ indicates significant $(p<0.05)$ differences from all other conditions; ns indicates no significant difference.

(van Groen and Wyss, 1990, 1992, 2003; Burwell and Amaral, 1998b) and because we wanted to directly compare the effects of the present lesions with those of RSP or POR that were performed using electrolytic methods in prior studies (Bucci et al., 2000; Keene and Bucci, 2008a). Control rats (SHAM; $n=7$ ) received surgery consisting of a craniotomy and shallow, nonpuncturing burr holes to minimize damage to underlying cortex. Rats were allowed to recover for $10-12 \mathrm{~d}$ before behavioral training.

\section{Behavioral apparatus and procedures}

IPSI, CONTRA, and SHAM rats were trained in the same fearconditioning task used previously to study the effects of bilateral damage to the RSP or POR (Bucci et al., 2000; Keene and Bucci, 2008a). During the training session, rats were placed in the chambers and permitted to explore the environment for $3 \mathrm{~min}$. Subsequently, three tone-shock trials separated by an ITI of $64 \mathrm{~s}$ were presented. During each trial, a $10 \mathrm{~s}$ tone stimulus $(1.5 \mathrm{kHz}, 78 \mathrm{~dB})$ was followed by delivery of a $1.0 \mathrm{~s}, 1.0 \mathrm{~mA}$ footshock. Rats were removed from the chambers $64 \mathrm{~s}$ after the last footshock. Twenty-four hours after the training session, rats were reexposed to the training chamber for a 10 min context test during which no tones or shocks were presented. Last, a tone test was conducted $24 \mathrm{~h}$ later by placing the rats in a novel context and presenting the tone 20 times beginning $30 \mathrm{~s}$ after the rat was placed in the chamber. Again, no shock was delivered during this test. The novel context consisted of the original training chambers outfitted with plain white paper on the walls of the chamber to hide the recessed food cup and other stimuli present on the aluminum walls. Cardboard was also placed on top of the grid floor to provide a different tactile stimulus, and a cup containing Vicks VapoRub and vinegar was placed in each sound-attenuating chamber to provide different olfactory cues. It has been shown previously that rats exhibit very little freezing behavior to the new context itself (Arenos et al., 2006). All rats received the context test first followed by the tone test since this has previously been shown to be the optimal method for obtaining the most independent assessment of both contextual and auditory fear conditioning in the same rats (Maren et al., 1997). Nevertheless, our laboratory has previously examined whether the order of testing impacts levels of freezing to the context and tone during the tests, and we have found identical results when the tone test was conducted before the context test (Arenos et al., 2006).

\section{Behavioral observations}

Scoring of freezing behavior that occurred during conditioning and during the context test was identical with that described in Experiment 1. For the tone test session, the incidence of freezing behavior was assessed every
$2 \mathrm{~s}$ during each $10 \mathrm{~s}$ presentation of the tone. The frequency of freezing behavior was converted to a percentage of total observations.

\section{Lesion verification}

After the behavioral procedures were completed, rats were deeply anesthetized with an overdose of Euthasol and transcardially perfused with $0.9 \%$ saline for $5 \mathrm{~min}$, followed by $10 \%$ buffered formalin. Brains were sectioned on a freezing microtome $(60 \mu \mathrm{m})$ and Nissl-stained using thionin. For each rat, coronal sections at six anteroposterior (AP) locations (from bregma in $\mathrm{mm}:-1.8,-3.3,-4.8,-6.3,-7.8$, and -8.3 ; see Fig. 6$)$ along the rostrocaudal extent of RSP and three locations (from bregma in $\mathrm{mm}:-7.8,-8.3$, -8.7 ) along the rostrocaudal extent of POR were used to assess the amount of tissue damage. Using StereoInvestigator software and a Zeiss Axioskope I compound microscope, gross tissue damage (necrosis, missing tissue, or marked thinning of tissue) was identified. For each coronal section, areal measurements were obtained using the StereoInvestigator Cavalieri estimator probe with $50 \mu \mathrm{m}$ grid spacing. The percentage damage to RSP and POR was calculated and any gross tissue damage to the area surrounding the target region was noted. In addition, the numbers of rats with and without damage to the cingulum bundle and postsubiculum were counted.

\section{Data analysis}

Analyses of freezing behavior that occurred during training and during the tone test were conducted using repeated-measures ANOVA with Group (CONTRA, IPSI, or SHAM) as the between-subjects variable and Trial as the within-subjects variable. For the context test data, repeatedmeasures ANOVA was conducted using Group as the between-subjects variable and Block (64 s epochs) as the within-subjects variable. An $\alpha$ value of 0.05 was adopted for all analyses, and post hoc analyses using Fisher's least significant difference test were used when appropriate.

\section{Results}

\section{Experiment 1: immediate-early gene expression in RSP during fear conditioning}

Behavioral confirmation of the immediate-shock deficit effect

The freezing behavior displayed by rats in the paired group during training is shown in Figure $2 \mathrm{~B}$. A repeated-measures ANOVA revealed a significant main effect of Trial $\left(F_{(3,18)}=44.9 ; p<0.0001\right)$, indicating that rats in the paired group acquired the fear response across trials. Analysis of the context test data revealed significant main effects of Group $\left(F_{(1,13)}=16.2 ; p<0.001\right)$ and 

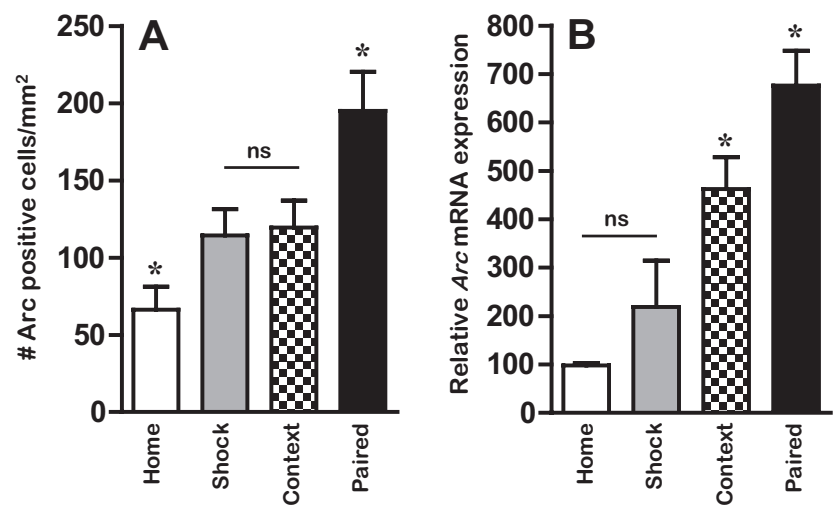

Figure 3. Arc expression in RSP following contextual fear conditioning. $A$, Average (mean \pm SEM) Arc protein expression in RSP across behavioral conditions. $n=4$ /group. $\boldsymbol{B}$, Relative $\operatorname{Arc}$ mRNA expression in RSP across behavioral conditions. Home $(n=6)$; Shock $(n=6)$; Context $(n=5)$; Paired $(n=7)$. The asterisk $\left(^{*}\right)$ indicates significant $(p<0.05)$ differences from all other conditions; ns indicates no significant difference. induction of nuclear c-Fos expression in RSP was observed across all behavioral conditions and varied as a function of Group $\left(F_{(3,22)}=9.2 ; p<0.0001\right.$; Fig. $\left.2 E\right)$. The number of nuclei immunoreactive for c-Fos in the paired group was significantly higher compared with all other groups (values of $p<0.05$ ). In addition, the number of c-Fos-positive nuclei in the context-only group was significantly different from all other groups (values of $p<0.05$ ), whereas the number of c-Fos-positive nuclei in the home cage and shock-only conditions did not differ from each other $(p>0.05)$. Thus, a stair step pattern of c-Fos expression in RSP was observed with paired $>$ context-only $>$ shock-only and home cage groups. Photomicrographs depicting c-Fos expression in RSP from rats in each condition are shown in Figure $2 F-I$.

Arc protein expression was also observed across all behavioral conditions and varied as a function of $\operatorname{Group}\left(F_{(3,12)}=9.3 ; p<0.05\right.$; Fig. 3A). The number of cells immunoreactive for Arc in the paired group was significantly higher compared with all other groups (values of $p<0.05$ ), whereas the number of cells immunoreactive for Arc was significantly lower in the home cage group compared with all other groups. In addition, the number of Arc-positive nuclei in the shock-only and
A

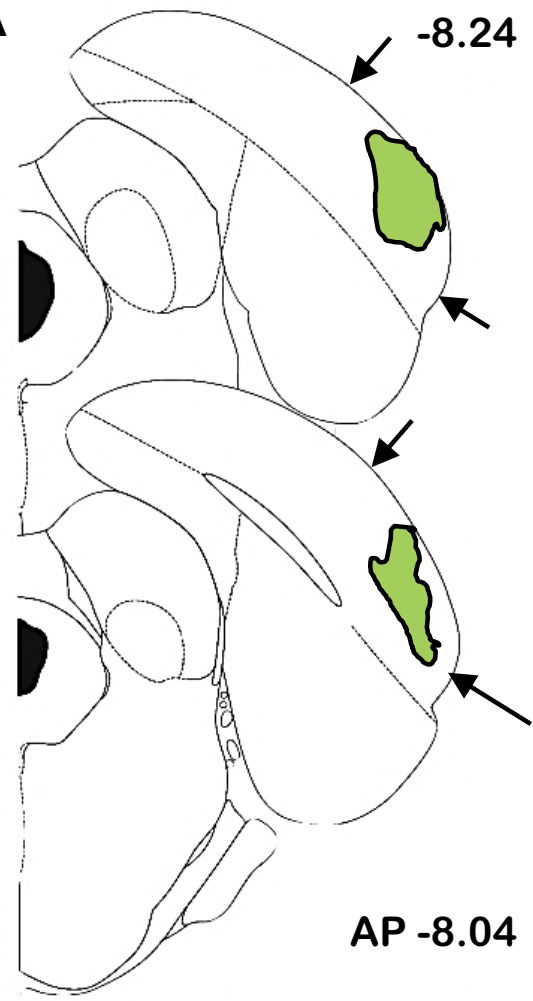

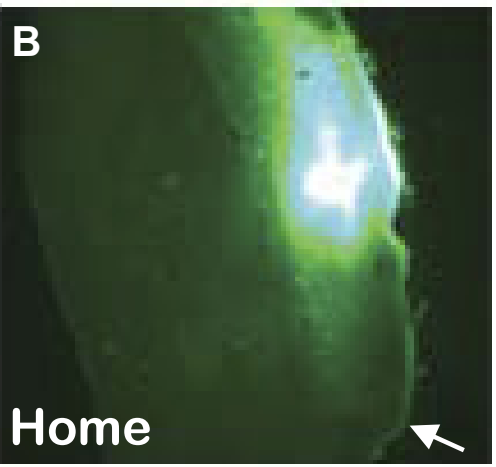

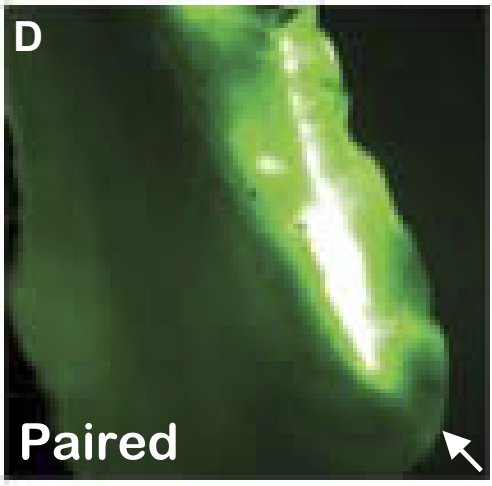

Figure 4. Representative images of the placement of infusions of the retrograde tracer CTb-488 into POR. A, C, Brain diagrams depicting infusion locations within POR. The AP location is noted to the right of the brain diagrams. $B, D$, Images of the fluorescent label indicating correct POR placement. The black arrows in $A$ and $C$ mark the dorsoventral boundaries of POR, and the white arrows in $\boldsymbol{B}$ and $\boldsymbol{D}$ mark the ventral boundary of POR.
Block $\left(F_{(9,117)}=3.0 ; p<0.05\right)$, but no Group by Block interaction $\left(F_{(9,117)}=1.9 ; p>0.05\right)$. As shown in Figure $2 C$, rats in the shockonly group exhibited near-zero levels of context-specific freezing compared with rats in the paired group, confirming that a contextshock association was not formed by rats in the shock-only group.

Immediate-early gene protein expression in RSP

The rat brain diagrams in Figure $2 D$ depict the AP locations from which c-Fos counts from RSP (shaded regions) were made. The context-only conditions did not differ from each other. The slightly different pattern of expression across groups compared with the c-Fos IHC data may be due to the lower $n$ (4 rats/group) in the Arc assay.

Arc $m R N A$ expression in RSP

The findings from the Arc mRNA qRT-PCR fear-conditioning experiment are presented in Figure 3B. Relative Arc mRNA expression was detected across all behavioral conditions and varied as a function of Group $\left(F_{(3,20)}=13.7 ; p<0.0001\right)$ with expression being significantly higher in the paired group compared with all other groups (values of $p<0.05$ ). In addition, relative Arc mRNA expression in the context-only group differed significantly from all other groups (values of $p<0.05$ ), whereas relative Arc mRNA expression in the home cage and shock-only condition did not differ from each other. This pattern of expression is the same as that observed in the c-Fos IHC assay.

\section{Experiment 2: activation of neurons that project from RSP to POR during fear conditioning Retrograde labeling}

Figure 4 depicts the size and location of representative microinfusions of the retrograde tracer, $\mathrm{CTb}-488$, into $\mathrm{POR}$. CTb-488 was accurately placed into the POR in 8 of 10 rats; thus, cell counts and identification of colocalized fluorescence labeling was quantified in 5 rats in the paired group and 3 rats in the home cage group. In addition, consistent with literature that describes the afferent innervation of POR (Burwell and Amaral, 1998a,b; Furtak et al., 2007), retrogradely labeled neurons were identified in the ipsilateral lateroposterior thalamic nucleus, perirhinal cortex, and visual cortex in all eight subjects included in the analysis (data not shown). 

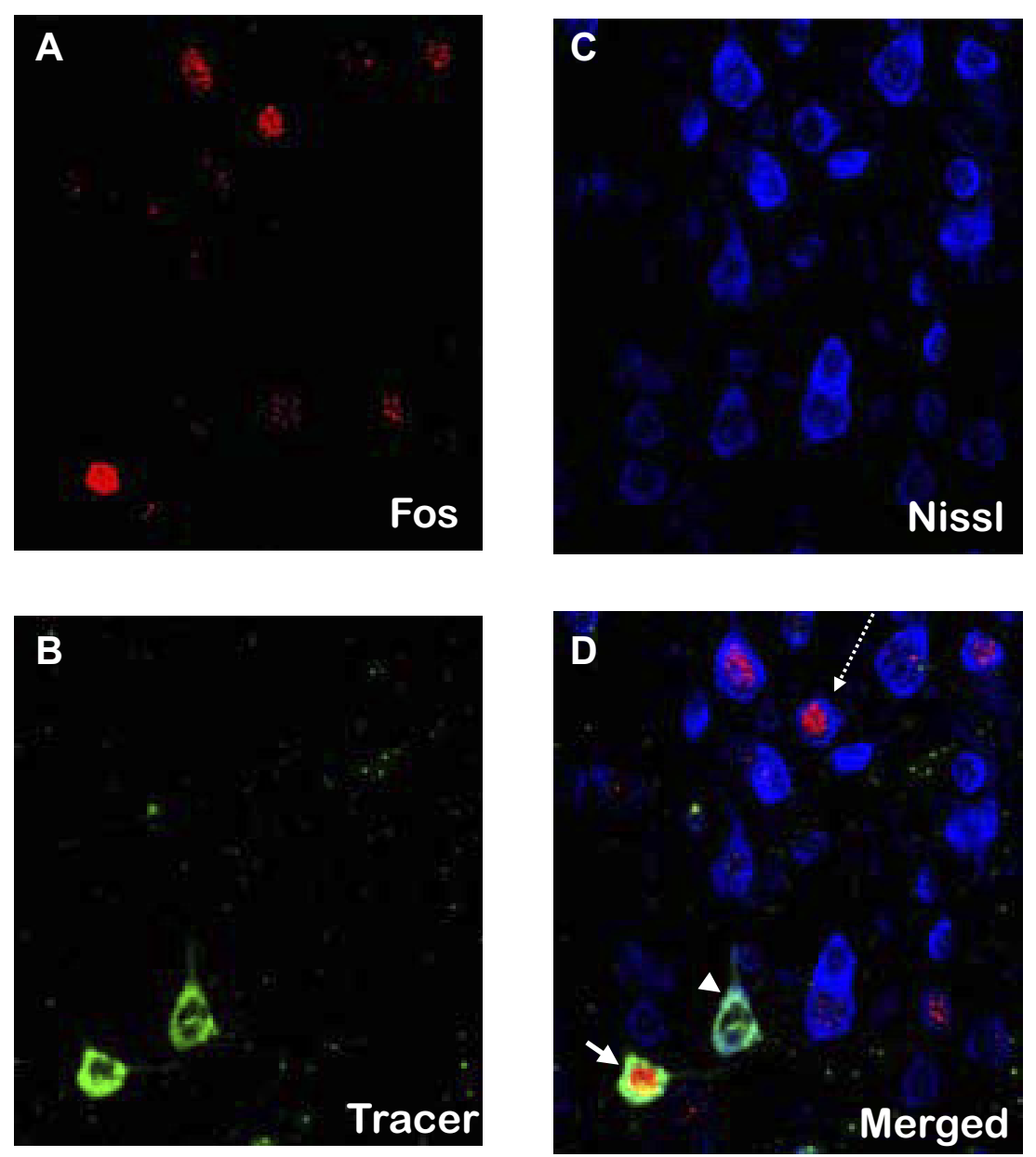

E

AP -6.8

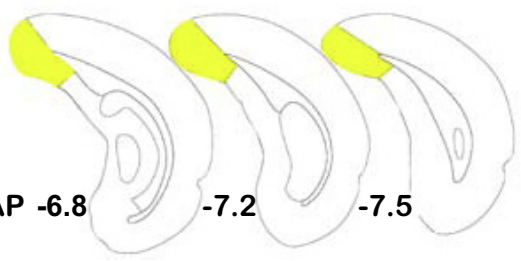

$\mathbf{F}$

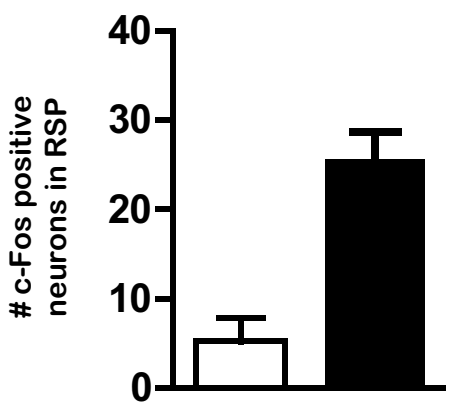

G

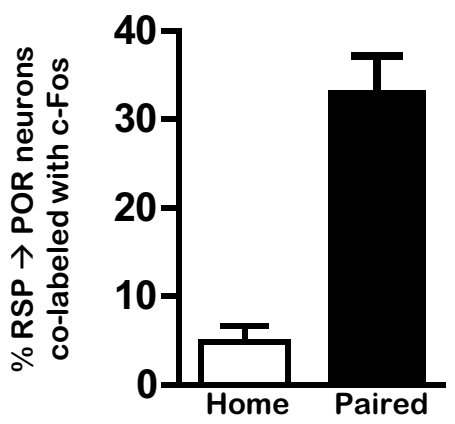

Figure 5. Histochemically verified neurons that project from RSP to POR are activated following fear conditioning. Photomicrographs of dorsal RSP neurons (ipsilateral to the injection site) obtained from sequential scans with a confocal microscope equipped with lasers to detect the c-Fos (A), the (Tb-488 tracer (B), and the Nissl labels (C). D, Merged image of the three labels depicted in $A-C$. The white arrow indicates a neuron (green) that projects from RSP to POR that is immunopositive for c-Fos (red), whereas the projection neuron (green) identified by the white arrowhead is not c-Fos positive. The arrow with the dashed line points to a Nissl-stained RSP cell (blue) that is immunopositive for c-Fos (red). This neuron (blue) was not retrogradely labeled, indicating that it does not project to POR. E, Brain diagrams reflecting the rostrocaudal location from which the confocal images of RSP neurons were collected. $\boldsymbol{F}$, Average (mean \pm SEM) c-Fos expression in RSP in home cage $(n=3)$ compared with fear-conditioned $(n=5)$ rats. G, Average (mean \pm SEM) percentage of RSP neurons that project to POR that are immunopositive for c-FoS.

c-Fos protein expression and colocalization of CTb-488 and c-Fos in RSP

Confocal microscopy was used to image cells in RSP that were fluorescently labeled with the retrograde tracer, $\mathrm{CTb}-488$, with a fluorescent secondary antibody to c-Fos and/or with a fluorescent Nissl stain. A representative montage of single and colabeled RSP cells from a fear-conditioned rat is presented in Figure $5 A-D$. Imaging revealed RSP neurons that project to POR (green label), RSP neurons that did not project to POR (blue label, Nisslstained neurons), and nuclei that were immunopositive for c-Fos (red label). The brain diagrams in Figure $5 E$ illustrate the regions of caudal RSP that were imaged. Quantification of the number of c-Fos-positive nuclei in RSP revealed robust c-Fos expression in rats in the paired group compared with home cage controls $\left(t_{(6)}=\right.$ -3.8 ; $p<0.05$; Fig. $5 F)$, an outcome that is similar to that observed in rostral RSP in Experiment 1. Furthermore, the percentage of cells that were colabeled with tracer and c-Fos immunofluorescence was significantly greater in paired compared with home cage rats $\left(t_{(6)}=-4.6\right.$; $p<0.05$; Fig. $\left.5 G\right)$, indicating that the corticocortical pathway that projects from RSP to POR was activated by the fear-conditioning procedure.

\section{Experiment 3: functional disconnection of RSP and POR during fear conditioning}

Histology

Five of 14 rats in the IPSI group and four of 11 rats in the CONTRA group were excluded from the statistical analyses due to incorrect lesion placement. Outlines of the largest and smallest of the ipsilateral $(n=9)$ and contralateral $(n=7)$ lesions are shown in Figure 6, $A$ and $B$, respectively. In the CONTRA group, the average area of unilateral RSP damage on each section analyzed was $70.1 \pm 6.9 \%$, and the average area of unilateral POR damage was $46.7 \pm 5.4 \%$. In the IPSI group, the average area of unilateral RSP damage on each section analyzed was $66.1 \pm 5.9 \%$, and the average area of unilateral POR damage was $61.5 \pm 7.8 \%$. Unilateral damage to the postsubiculum was observed in three of seven rats in the CONTRA group and in five of nine rats in the IPSI group. Minor damage to the cingulum bundle was observed in three of seven rats in the CONTRA group and in six of nine rats in the IPSI group.

\section{Fear conditioning}

Figure $7 A$ illustrates that rats in each group (CONTRA, IPSI, or SHAM) exhibited robust postshock freezing during the ac- 


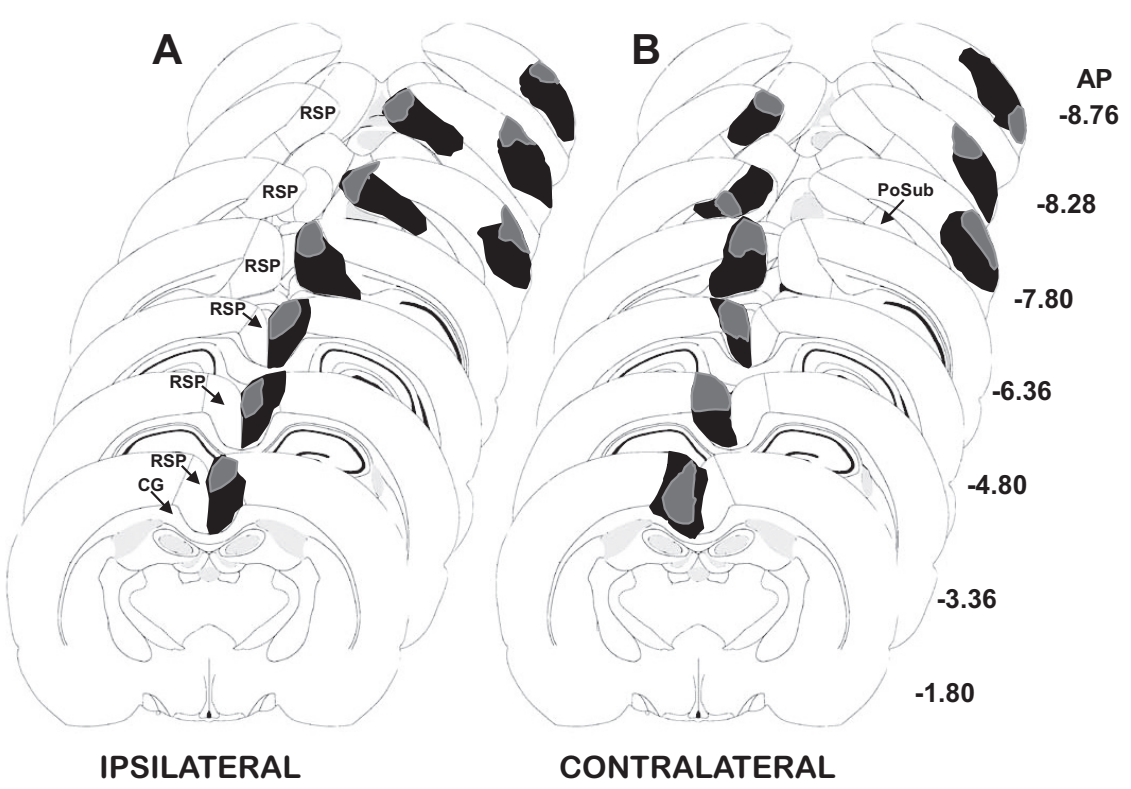

Figure 6. Collages of brain diagrams along the rostrocaudal extent of the RSP and POR indicating the largest (black) and smallest (gray) ipsilateral $(\boldsymbol{A})$ and contralateral ( $\boldsymbol{B}$ ) unilateral lesions of the RSP and POR. CG, Cingulum bundle; PoSub, postsubiculum; RSP, retrosplenial cortex.
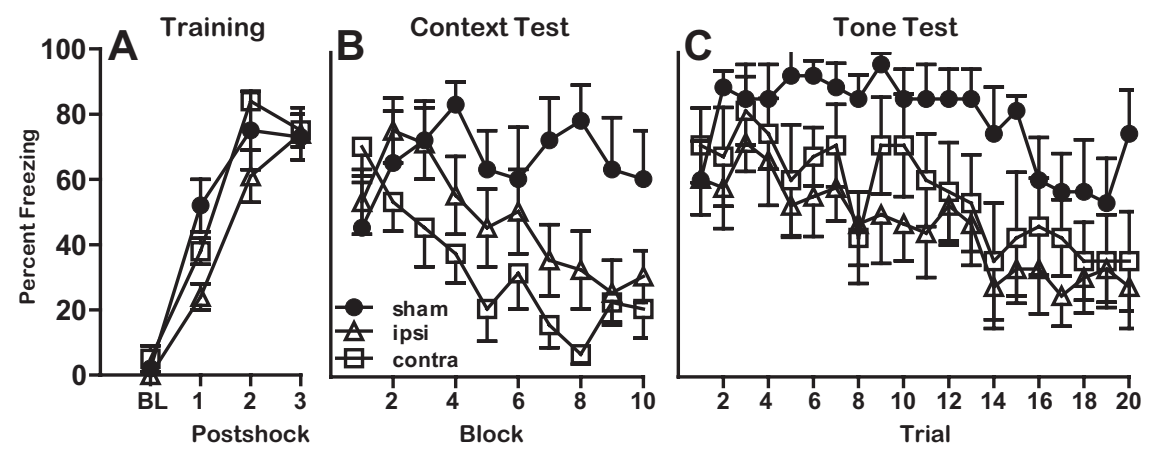

Figure 7. Freezing behavior during fear conditioning. Effects of unilateral RSP-POR lesions on the freezing behavior of ipsilateral (ipsi) $(n=9)$, contralateral (contra) $(n=7)$ or sham-operated (sham) $(n=7)$ rats during the Training session $(\boldsymbol{A})$ and during the Context $(\boldsymbol{B})$ and Tone $(\boldsymbol{C})$ tests.

quisition session. A repeated-measures ANOVA revealed a significant main effect of Trial $\left(F_{(3,60)}=124.7 ; p<0.0001\right)$, no main effect of Group $\left(F_{(2,20)}=2.5 ; p>0.05\right)$, and a trend toward a significant Group by Trial interaction $\left(F_{(6,60)}=2.2 ; p=0.055\right)$. Inspection of the postshock freezing curves revealed that all groups exhibited equivalent postshock freezing after the third shock, but that rats in the IPSI group tended to freeze less after the first and second shock compared with the CONTRA and SHAM conditions.

An analysis of the context test data revealed significant main effects of Group $\left(F_{(1,20)}=113.3 ; p<0.001\right)$ and Block $\left(F_{(9,180)}=\right.$ 5.1; $p<0.001)$ and a significant Group by Block interaction $\left(F_{(18,180)}=2.9 ; p<0.001\right)$. As shown in Figure $7 B$, rats in the CONTRA lesion group exhibited low levels of context-specific freezing compared with SHAM rats $(p<0.01)$. The level of context freezing in the IPSI group was between that of the other groups and did not differ significantly from either SHAM or CONTRA rats. If IPSI lesions had a strong effect on behavior, then the level of freezing in the IPSI rats should be significantly different from the SHAM group, but this effect was not observed. Similarly, if IPSI lesions were without effect on behavior, then the level of freezing displayed by rats in the IPSI group should be significantly less than rats in the CONTRA group, but this was not observed either. In summary, the data reveal that rats in the CONTRA group displayed the least amount of freezing, whereas the level of freezing displayed by the IPSI group was intermediate between the CONTRA and SHAM groups and rats in the SHAM group displayed the highest percentage of context-specific freezing.

Data from the tone test are presented in Figure 7C. Repeated-measures ANOVA revealed significant main effects of Group $\left(F_{(2,20)}=4.8 ; p<0.05\right)$ and Trial $\left(F_{(19,380)}\right.$ $=5.0 ; p<0.001)$ but no significant Group by Trial interaction $\left(F_{(38,380)}=0.7\right.$; $p>0.05)$. The initially high level of freezing in the SHAM group diminished over the course of the extinction session, whereas CONTRA-lesioned rats exhibited less freezing during the majority of the tone presentations compared with SHAMS $(p<0.01)$. Rats in the IPSI group displayed similar levels of freezing compared with the CONTRA group $(p>0.05)$ and a trend toward less freezing compared with the SHAM group $(p=0.06)$.

\section{Discussion}

Immediate-early gene expression in RSP during fear conditioning

The present set of experiments tested the involvement of RSP and its connections with POR in contextual fear learning/ memory using a combined analysis of neural activity, anatomical tracing, and lesion-disconnection methods. To date, while a role for RSP in contextual memory has been documented (Beck and Fibiger, 1995; Keene and Bucci, 2008c; Corcoran et al., 2011), there are no data that specifically examine whether RSP neurons are activated during acquisition (i.e., learning) of contextual fear conditioning. Experiment 1 addressed this gap in the literature by demonstrating a stair step pattern of c-Fos expression across behavioral conditions, with low c-Fos expression in the home cage and shock-only conditions, intermediate expression after exploration of the context, and the highest c-Fos expression observed in fear-conditioned rats. Like c-Fos, a stair step pattern of Arc mRNA expression was observed across behavioral conditions, which is consistent with studies reporting learning-related induction of Arc in hippocampus (Lonergan et al., 2010) and amygdala (Ploski et al., 2008) following fear conditioning. The context-specific elevation of IEG expression confirms previous reports of exploration-induced Arc expression in murine RSP (Spulber et al., 2009) and complements reports of increased Arc expression in hippocampus following rapid encoding of novel environments (Pevzner et al., 2012). One interpretation of the present data is that neurons in RSP actively participate in encoding two associative processes, one that involves the formation of associations between contextual stimuli (i.e., stimu- 
lus-stimulus associations) and one that links contextual cues with shock (i.e., stimulus-unconditioned stimulus associations).

Together, these data support the notion that RSP has a role in forming associations between sensory stimuli, consistent with lesion studies showing that permanent RSP damage impairs not only contextual fear conditioning (Keene and Bucci, 2008a,c) but also appetitive forms of relational learning (Keene and Bucci, 2008b; Robinson et al., 2011). Similarly, inactivation of RSP has previously been shown to impair hippocampal-dependent spatial learning (Cooper and Mizumori, 1999, 2001), and electrophysiological studies in rabbits indicate that neurons in the posterior cingulate cortex (comparable with RSP in rats) are active during training in tasks that require the formation of contextual associations (Gabriel, 1990; Freeman et al., 1996).

However, a role for RSP in learning is contradicted by a recent study of fear conditioning in mice (Corcoran et al., 2011). In that study, mice received infusions of an NMDA receptor antagonist into RSP before training and subsequently exhibited normal levels of freezing when reexposed to the training chamber (drug free), which the authors interpreted as evidence that RSP does not have a role in learning. However, it may be that NMDA receptors per se are not critical to RSP function during acquisition, and that the contributions to RSP in forming associations during this phase are reliant on other neurotransmitter receptors. In addition, the NMDA receptor antagonist was only infused into a single site in RSP, leaving much of the region unaffected [RSP extends for several millimeters in the rostrocaudal axis (Paxinos and Watson, 2007)]. A more definitive test of the involvement of RSP in learning per se would require infusion of agents that inactivate or inhibit all types of neurons, and the development of techniques to do so throughout the full rostral-caudal extent of the RSP.

\section{Functional connections between RSP and POR during fear conditioning}

A second main finding from the present studies is that a functional interaction exists between RSP and POR during contextual fear conditioning. Specifically, the results of the tracing study (Experiment 2) revealed that a proportion $(\sim 33 \%)$ of retrogradely labeled RSP neurons that project to POR are among those that are activated following fear conditioning. In contrast, in the home cage control rats, few $(\sim 5 \%)$ retrogradely labeled RSP neurons were c-Fos positive. Although these data suggest that RSP and POR communicate during fear conditioning, they do not address whether this interaction is necessary to support contextual fear learning/memory. This was more rigorously tested in Experiment 3 by disconnecting RSP and POR before fear conditioning. Unilateral RSP and POR lesions on the same side of the brain [thus leaving the connection between RSP and POR intact in one hemisphere (i.e., IPSI group)] had a modest effect on contextual fear memory, whereas rats with unilateral lesions of RSP and POR in contralateral hemispheres [resulting in a functional bilateral disconnection of RSP and POR (i.e., CONTRA group)] exhibited deficits in contextual freezing compared with sham-lesioned rats when reexposed to the training chamber. The deficits observed in the CONTRA group were comparable with those observed in previous studies in which bilateral lesions were made to either RSP or POR (Bucci et al., 2000; Keene and Bucci, $2008 \mathrm{a}, \mathrm{c})$. Importantly, these data indicate that connections between RSP and POR are necessary to support normal contextual fear conditioning and, together with the neural activation studies, suggest that RSP and POR are components of an integrated neural circuit that mediates fear conditioning.
A surprising result was that both ipsilateral and contralateral lesions produced reductions in the expression of auditory fear memory, an effect that was not observed in previous studies that examined bilateral lesions of either RSP or POR (Bucci et al., 2000; Keene and Bucci, 2008a,c). The difference in results may be due to the extent of damage to RSP, which was considerably larger in the present study ( $\sim 68 \%$ of RSP tissue was damaged in the present study compared with $\sim 40 \%$ in prior studies that also used the electrolytic approach). It is conceivable that a similarly large percentage of bilateral RSP and/or POR damage would lead to auditory fear deficits like those observed in the present study. Indeed, Radwanka et al. (2010) recently demonstrated that IEG expression and glucose utilization in RSP is elevated following single-cue fear conditioning. Furthermore, as a consequence of the greater extent of lesion damage in the present study, some unilateral damage to the postsubiculum was observed in three of seven rats with contralateral lesions and five of nine rats with ipsilateral lesions. Damage to postsubiculum has been shown previously to affect the acquisition and expression of auditory fear conditioning (Robinson and Bucci, 2011). Thus, it is possible that unintentional damage to PoSub contributed to the deficits observed in the present study. A third explanation, which is not mutually exclusive from those proposed above, is that the mild deficit in postshock freezing expressed by rats with ipsilateral lesions may have contributed to low expression of auditory fear memory by this group. It is noteworthy that, while the majority of hippocampal lesion studies report context, but not acquisition or auditory fear memory deficits, there are examples to the contrary (Maren and Holt, 2004). Thus, it appears that, under specific conditions, RSP and/or POR may indeed contribute to both auditory and contextual fear memory. Finally, while it is possible that damage to RSP or POR might have impaired processing of the footshock itself, this is unlikely since rats with bilateral RSP or POR damage in prior reports (Bucci et al., 2000; Keene and Bucci, 2008a,c) exhibited normal acquisition of the fear response during training and normal tone fear memory.

\section{Putative roles for RSP and POR during fear conditioning}

The rationale for investigating the projection from RSP to POR was based on theoretical, behavioral, and anatomical research that supports roles for both regions during contextual information processing. Because RSP and POR have in common a number of inputs and outputs, it is possible that these regions perform redundant functions during fear conditioning. However, arguing against this possibility are the many distinct connections with cortical and subcortical structures for each region (for review, see Burwell, 2000; Furtak et al., 2007). Although speculative, perhaps RSP contributes to fear conditioning by facilitating the formation of associations among environmental conditioned and/or unconditioned stimuli (Keene and Bucci, 2008b; Radwanska et al., 2010; Robinson et al., 2011; Tse et al., 2011), whereas POR contributes by monitoring changes in environmental stimuli (Burwell and Hafeman, 2003; Bucci and Burwell, 2004; Sills et al., 2012). Yet another possibility is that before forwarding sensory information to hippocampus, rhinal cortex serves to filter nonrelevant stimuli through GABAergic inhibitory mechanisms (de Curtis and Paré, 2004), a notion that has recently been further validated by a study that characterized the electrophysiological properties of POR pyramidal cells and interneurons (Sills et al., 2012).

Collectively, the methods used in the present study provide information about the functional anatomy of this circuitry that extends beyond those garnered from the results of lesion studies 
alone. Specifically, the IEG data from Experiment 1 provide direct evidence that RSP is indeed engaged during the time that context-specific learning is occurring. Furthermore, a significant proportion of RSP neurons with histologically verified connections to POR were activated following exposure to a fearconditioning paradigm, demonstrating a behaviorally relevant increase in activity within this pathway. Last, the disconnection study provides the first evidence of a necessary functional relationship between RSP and POR in fear conditioning. Additional studies which use multiple distinguishable retrograde tracers in combination with immunohistochemical techniques will further elucidate the corticohippocampal circuitry that underlies fear conditioning.

\section{References}

Aggleton JP (2011) Multiple anatomical systems embedded within the primate medial temporal lobe: implications for hippocampal function. Neurosci Biobehav Rev. Advance online publication. Retrieved July 24, 2012. doi:10.1016/j.neubiorev.2011.09.005.

Arenos JD, Musty RE, Bucci DJ (2006) Blockade of cannabinoid CB1 receptors alters contextual learning and memory. Eur J Pharmacol 539:177-183.

Bar M, Aminoff E (2003) Cortical analysis of visual context. Neuron 38:347-358.

Beck CH, Fibiger HC (1995) Conditioned fear-induced changes in behavior and in the expression of the immediate early gene c-fos: with and without diazepam pretreatment. J Neurosci 15:709-720.

Blanchard RJ, Blanchard DC (1969) Crouching as an index of fear. J Comp Physiol Psychol 67:370-375.

Bucci DJ, Burwell RD (2004) Deficits in attentional orienting following damage to the perirhinal or postrhinal cortices. Behav Neurosci 118: $1117-1122$.

Bucci DJ, Phillips RG, Burwell RD (2000) Contributions of postrhinal and perirhinal cortex to contextual information processing. Behav Neurosci 114:882-894.

Burgess N (2008) Spatial cognition and the brain. Ann N Y Acad Sci 1124:77-97.

Burwell RD (2000) The parahippocampal region: corticocortical connectivity. Ann N Y Acad Sci 911:25-42.

Burwell RD, Amaral DG (1998a) Perirhinal and postrhinal cortices of the rat: interconnectivity and connections with the entorhinal cortex. J Comp Neurol 391:293-321.

Burwell RD, Amaral DG (1998b) Cortical afferents of the perirhinal, postrhinal, and entorhinal cortices of the rat. J Comp Neurol 398:179-205.

Burwell RD, Hafeman DM (2003) Positional firing properties of postrhinal cortex neurons. Neuroscience 119:577-588.

Campeau S, Hayward MD, Hope BT, Rosen JB, Nestler EJ, Davis M (1991) Induction of the c-fos proto-oncogene in rat amygdala during unconditioned and conditioned fear. Brain Res 565:349-352.

Conte WL, Kamishina H, Reep RL (2009) Multiple neuroanatomical tracttracing using fluorescent Alexa Fluor conjugates of cholera toxin subunit B in rats. Nat Protoc 4:1157-1166.

Cooper BG, Mizumori SJ (1999) Retrosplenial cortex inactivation selectively impairs navigation in darkness. Neuroreport 10:625-630.

Cooper BG, Mizumori SJ (2001) Temporary inactivation of the retrosplenial cortex causes a transient reorganization of spatial coding in the hippocampus. J Neurosci 21:3986-4001.

Corcoran KA, Donnan MD, Tronson NC, Guzmán YF, Gao C, Jovasevic V, Guedea AL, Radulovic J (2011) NMDA receptors in retrosplenial cortex are necessary for retrieval of recent and remote context fear memory. J Neurosci 31:11655-11659.

de Curtis M, Paré D (2004) The rhinal cortices: a wall of inhibition between the neocortex and the hippocampus. Prog Neurobiol 74:101-110.

Eichenbaum H, Sauvage M, Fortin N, Komorowski R, Lipton P (2011) Towards a functional organization of episodic memory in the medial temporal lobe. Neurosci Biobehav Rev. Advance online publication. Retrieved July 24, 2012. doi:10.1016/j.neubiorev.2011.07.006.

Fanselow MS (1980) Conditioned and unconditional components of postshock freezing. Pavlov J Biol Sci 15:177-182.
Fanselow MS (1986) Associative vs topographical accounts of the immediate shock-freezing deficit in rats: implications for the response selection rules governing species-specific defensive reactions. Learn Motiv 17:16-39.

Freeman JH Jr, Cuppernell C, Flannery K, Gabriel M (1996) Limbic thalamic, cingulate cortical and hippocampal neuronal correlates of discriminative approach learning in rabbits. Behav Brain Res 80:123-136.

Furtak SC, Wei SM, Agster KL, Burwell RD (2007) Functional neuroanatomy of the parahippocampal region in the rat: the perirhinal and postrhinal cortices. Hippocampus 17:709-722.

Gabriel M (1990) Functions of anterior and posterior cingulate cortex during avoidance learning in rabbits. Prog Brain Res 85:467-482; discussion $482-483$.

Guzowski JF, McNaughton BL, Barnes CA, Worley PF (1999) Environmentspecific expression of the immediate-early gene arc in hippocampal neuronal ensembles. Nat Neurosci 2:1120-1124.

Harker KT, Whishaw IQ (2004) Impaired place navigation in place and matching-to-place swimming pool tasks follows both retrosplenial cortex lesions and cingulum bundle lesions in rats. Hippocampus 14:224-231.

Holland PC, Bouton ME (1999) Hippocampus and context in classical conditioning. Curr Opin Neurobiol 9:195-202.

Hughes P, Dragunow M (1994) Activation of pirenzepine-sensitive muscarinic receptors induces a specific pattern of immediate-early gene expression in rat brain neurons. Brain Res Mol Brain Res 24:166-178.

Jones BF, Witter MP (2007) Cingulate cortex projections to the parahippocampal region and hippocampal formation in the rat. Hippocampus 17:957-976.

Keene CS, Bucci DJ (2008a) Contributions of the retrosplenial and posterior parietal cortices to cue-specific and contextual fear conditioning. Behav Neurosci 122:89-97.

Keene CS, Bucci DJ (2008b) Involvement of the retrosplenial cortex in processing multiple conditioned stimuli. Behav Neurosci 122:651-658.

Keene CS, Bucci DJ (2008c) Neurotoxic lesions of retrosplenial cortex disrupt signaled and unsignaled contextual fear conditioning. Behav Neurosci 122:1070-1077.

Kobayashi Y, Amaral DG (2007) Macaque monkey retrosplenial cortex: III. Cortical efferents. J Comp Neurol 502:810-833.

Kubik S, Miyashita T, Kubik-Zahorodna A, Guzowski JF (2012) Loss of activity-dependent arc gene expression in the retrosplenial cortex after hippocampal inactivation: interaction in a higher-order memory circuit. Neurobiol Learn Mem 97:124-131.

Lonergan ME, Gafford GM, Jarome TJ, Helmstetter FJ (2010) Timedependent expression of arc and zif268 after acquisition of fear conditioning. Neural Plast 2010:139891.

Maren S, Fanselow MS (1997) Electrolytic lesions of the fimbria/fornix, dorsal hippocampus, or entorhinal cortex produce anterograde deficits in contextual fear conditioning in rats. Neurobiol Learn Mem 67:142-149.

Maren S, Holt WG (2004) Hippocampus and Pavlovian fear conditioning in rats: muscimol infusions into the ventral, but not dorsal, hippocampus impair the acquisition of conditional freezing to an auditory conditional stimulus. Behav Neurosci 118:97-110.

Maren S, Aharonov G, Fanselow MS (1997) Neurotoxic lesions of the dorsal hippocampus and Pavlovian fear conditioning in rats. Behav Brain Res $88: 261-274$.

Maren S, Anagnostaras SG, Fanselow MS (1998) The startled seahorse: is the hippocampus necessary for contextual fear conditioning? Trends Cogn Sci 2:39-42.

Matsui A, Williams JT (2010) Activation of micro-opioid receptors and block of Kir3 potassium channels and NMDA receptor conductance by Land D-methadone in rat locus coeruleus. Br J Pharmacol 161:1403-1413.

Miyashita T, Kubik S, Haghighi N, Steward O, Guzowski JF (2009) Rapid activation of plasticity-associated gene transcription in hippocampal neurons provides a mechanism for encoding of one-trial experience. J Neurosci 29:898-906.

Olton DS (1983) The use of animal models to evaluate the effects of neurotoxins on cognitive processes. Neurobehav Toxicol Teratol 5:635-640.

Paxinos G, Watson C (2007) The rat brain in stereotaxic coordinates, Ed 6 . San Diego: Elsevier Academic.

Pevzner A, Miyashita T, Schiffman AJ, Guzowski JF (2012) Temporal dynamics of arc gene induction in hippocampus: relationship to context memory formation. Neurobiol Learn Mem 97:313-320.

Pezzone MA, Lee WS, Hoffman GE, Rabin BS (1992) Induction of c-Fos 
immunoreactivity in the rat forebrain by conditioned and unconditioned aversive stimuli. Brain Res 597:41-50.

Ploski JE, Pierre VJ, Smucny J, Park K, Monsey MS, Overeem KA, Schafe GE (2008) The activity-regulated cytoskeletal-associated protein (Arc/ Arg3.1) is required for memory consolidation of Pavlovian fear conditioning in the lateral amygdala. J Neurosci 28:12383-12395.

Radwanska A, Debowska W, Liguz-Lecznar M, Brzezicka A, Kossut M, Cybulska-Klosowicz A (2010) Involvement of retrosplenial cortex in classical conditioning. Behav Brain Res 214:231-239.

Robinson S, Keene CS, Iaccarino HF, Duan D, Bucci DJ (2011) Involvement of retrosplenial cortex in forming associations between multiple sensory stimuli. Behav Neurosci 125:578-587.

Rogers JL, Kesner RP (2007) Hippocampal-parietal cortex interactions: evidence from a disconnection study in the rat. Behav Brain Res 179:19-27.

Shepherd JD, Bear MF (2011) New views of Arc, a master regulator of synaptic plasticity. Nat Neurosci 14:279-284.

Sills JB, Connors BW, Burwell RD (2012) Electrophysiological and morphological properties of neurons in layer 5 of the rat postrhinal cortex. Hippocampus. Advance online publication. Retrieved July 24, 2012. doi:10.1002/hipo.22026.

Smith DM, Wakeman D, Patel J, Gabriel M (2004) Fornix lesions impair context-related cingulothalamic neuronal patterns and concurrent discrimination learning in rabbits (Oryctolagus cuniculus). Behav Neurosci 118:1225-1239.

Spulber S, Mateos L, Oprica M, Cedazo-Minguez A, Bartfai T, Winblad B, Schultzberg M (2009) Impaired long term memory consolidation in transgenic mice overexpressing the human soluble form of IL-1ra in the brain. J Neuroimmunol 208:46-53.

Staresina BP, Duncan KD, Davachi L (2011) Perirhinal and parahippocampal cortices differentially contribute to later recollection of object- and scene-related event details. J Neurosci 31:8739-8747.
Sugar J, Witter MP, van Strien NM, Cappaert NL (2011) The retrosplenial cortex: intrinsic connectivity and connections with the parahippocampal region in the rat. An interactive connectome. Front Neuroinform 5:7.

Talk A, Stoll E, Gabriel M (2005) Cingulate cortical coding of contextdependent latent inhibition. Behav Neurosci 119:1524-1532.

Tse D, Takeuchi T, Kakeyama M, Kajii Y, Okuno H, Tohyama C, Bito H, Morris RG (2011) Schema-dependent gene activation and memory encoding in neocortex. Science 333:891-895.

van Groen T, Wyss JM (1990) Connections of the retrosplenial granular a cortex in the rat. J Comp Neurol 300:593-606.

van Groen T, Wyss JM (1992) Connections of the retrosplenial dysgranular cortex in the rat. J Comp Neurol 315:200-216.

van Groen T, Wyss JM (2003) Connections of the retrosplenial granular b cortex in the rat. J Comp Neurol 463:249-263.

Vann SD, Aggleton JP, Maguire EA (2009) What does the retrosplenial cortex do? Nat Rev Neurosci 10:792-802.

van Strien NM, Cappaert NL, Witter MP (2009) The anatomy of memory: an interactive overview of the parahippocampal-hippocampal network. Nat Rev Neurosci 10:272-282.

Vazdarjanova A, Ramirez-Amaya V, Insel N, Plummer TK, Rosi S, Chowdhury S, Mikhael D, Worley PF, Guzowski JF, Barnes CA (2006) Spatial exploration induces ARC, a plasticity-related immediate-early gene, only in calcium/calmodulin-dependent protein kinase II-positive principal excitatory and inhibitory neurons of the rat forebrain. J Comp Neurol 498:317-329.

Warburton EC, Baird A, Morgan A, Muir JL, Aggleton JP (2001) The conjoint importance of the hippocampus and anterior thalamic nuclei for allocentric spatial learning: evidence from a disconnection study in the rat. J Neurosci 21:7323-7330.

Wixted JT, Squire LR (2011) The medial temporal lobe and the attributes of memory. Trends Cogn Sci 15:210-217. 